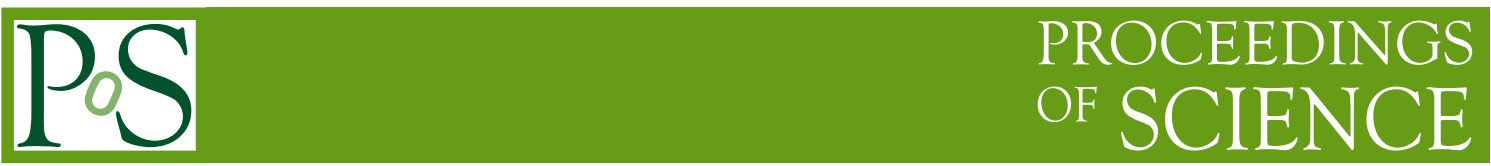

\title{
Experimental beam tests for FCC-ee
}

\author{
J. Keintzel, ${ }^{a, *}$ M. Benedikt, R. Tomás and F. Zimmermann \\ On behalf of the FCC-ee collaboration \\ CERN, 1211 Geneva 23, Switzerland \\ a also at TU Wien, 1040 Vienna, Austria \\ E-mail: jacqueline.keintzel@cern.ch, michael.benedikt@cern.ch, \\ rogelio.tomas@cern.ch, frank.zimmermann@cern.ch
}

\begin{abstract}
Possible circular colliders for the post-LHC era at CERN are being explored within the framework of the Future Circular Collider (FCC) feasibility study. The first stage of the FCC integrated project is the FCC-ee, an ambitious electron-positron collider with a circumference of approximately $100 \mathrm{~km}$. Certain key concepts of the FCC-ee design can be demonstrated and tested at existing facilities, such as at SuperKEKB at KEK, DAФNE at INFN, KARA at KIT, PETRA III at DESY, VEPP-4M at BINP, and the SwissFEL at PSI. The test of a prototype positron source at PSI, understanding the crab-waist collision scheme at SuperKEKB and DAФNE, testing optics control and emittance tuning techniques at SuperKEKB and PETRA III, and precise beam energy calibration at VEPP-4M will offer invaluable insights for the FCC-ee design optimization and its operational procedures. The results of these beam tests will be an essential input to the FCC Feasibility Study Report. This article will give an overview of possible experimental tests for FCC-ee and highlight already successfully performed studies.
\end{abstract}

\footnotetext{
*** The European Physical Society Conference on High Energy Physics (EPS-HEP2021), ***

*** 26-30 July $2021 * * *$

*** Online conference, jointly organized by Universität Hamburg and the research center DESY ***
}

\footnotetext{
${ }^{*}$ Speaker
} 


\section{Introduction}

The Large Hadron Collider (LHC) [1] at CERN is presently the largest circular collider with a circumference of almost $27 \mathrm{~km}$ and has successfully collided two counter-rotating proton beams with a beam energy of $6.5 \mathrm{TeV}$ in the past run, while providing a peak instantaneous luminosity of $2 \times 10^{34} \mathrm{~cm}^{-2} \mathrm{~s}^{-1}$. Its luminosity upgrade, the High Luminosity (HL-LHC) [2], is scheduled to start operating around 2027 with a beam energy of $7 \mathrm{TeV}$ and a design instantaneous levelled luminosity of about $5 \times 10^{34} \mathrm{~cm}^{-2} \mathrm{~s}^{-1}$.

In the framework of the Future Circular Collider (FCC) feasibility study [3] possible lepton and hadron circular colliders are being explored for the post-HL-LHC era. The FCC integrated project [4] foresees first the installation and commissioning of an electron-positron collider, the FCC-ee [5], followed by a hadron collider, the FCC-hh [6], where the latter aims to achieve a beam energy of $50 \mathrm{TeV}$. As both colliders would be installed in the same tunnel infrastructure, they demand a similar footprint including the circumference of almost $100 \mathrm{~km}$. The commissioning of the lepton and the hadron collider is foreseen around 2040 and 2065, respectively. Although the fist stage of the integrated FCC project would be commissioned in about 20 years, certain key concepts could already be tested before its construction at existing facilities. Results obtained from dedicated beam tests will probe concepts of the FCC-ee design and will also be included in the FCC feasibility study report.

\section{A brief description of the FCC-ee}

The FCC-ee is designed to collide one electron with one positron beam at four different beam energies of $45.6 \mathrm{GeV}, 80 \mathrm{GeV}, 120 \mathrm{GeV}$ and $185.5 \mathrm{GeV}$, thus, demanding a flexible lattice and optics configuration, where selected FCC-ee parameters are given in Table 1 [5]. While the first two stages are dedicated to studying the $\mathrm{W}$ - and Z-bosons, the third energy stage focuses on the Higgs-boson. Colliding beams at the highest energy allows studying physics related to the top quark. In the conceptual design report two crossing points for high energy physics experiments are foreseen, while recent investigations [7] study the feasibility of four interaction regions dedicated to high energy physics experiments. To reach the design values tight optics and alignment tolerances need to be respected for all stages. The highest peak luminosity of $230 \times 10^{34} \mathrm{~cm}^{-2} \mathrm{~s}^{-1}$ is planned for the lowest energy, achieved by applying the crab-waist collision scheme and operating high beam currents of up to almost 1.4 A. The latter also demands an innovative positron source for their production and the integration in the lattice. High luminosity production, however, also results in a

\begin{tabular}{lcccc}
\hline \hline Parameter & Z & WW & ZH & ttbar \\
\hline Beam energy [GeV] & 45.6 & 80 & 120 & 182.5 \\
Beam current [mA] & 1390 & 147 & 29 & 5.4 \\
$\beta_{x, y}^{*}[\mathrm{~cm}]$ & $15 / 0.08$ & $20 / 0.1$ & $30 / 0.1$ & $100 / 0.16$ \\
Bunch length with SR/BS [mm] & $3.5 / 12.1$ & $3.0 / 6.0$ & $3.15 / 5.3$ & $1.97 / 2.54$ \\
Luminosity $\left[10^{34} \mathrm{~cm}^{-2} \mathrm{~s}^{-1}\right]$ & 230 & 28 & 8.5 & 1.55 \\
\hline \hline
\end{tabular}

Table 1: Selected FCC-ee beam parameters [5]. 
short beam lifetime. To compensate for particle losses a continuous beam injection from the FCC-ee booster [5, 8] into the main rings is foreseen, known as top-up injection. The booster is also designed to be housed in the same tunnel infrastructure and hence features a similar footprint. One strong motivation for the FCC-ee is that it allows for high precision high energy physics experiments [9], demanding the determination of the exact collision energy. Since the FCC-ee is designed to collide polarized beams, resonant depolarization is envisaged to be used for energy calibration.

\section{Beam tests at existing facilities}

Numerous design concepts of the FCC-ee are applied in existing facilities. In addition, novel components for the FCC-ee are also envisaged to be tested, helping to validate their design choices.

At the first FCC-ee stage with almost 1.4 A beam current, each of the 16640 bunches consists of about $2 \times 10^{10}$ electrons or positrons [5]. The latter are produced by sending the electron beam onto a target, where ongoing studies investigate in the most suitable design. While a conventional target consists of a material with a high atomic number and melting point, a hybrid target combined with a flux concentrator could be optimized to reduce the peak energy deposition [10]. Dedicated studies at the Swiss x-ray Free Electron Laser (SwissFEL) [11], located at PSI, could help investigating the most suitable target scheme and also test the feasibility of using solenoids for positron capture.

The first machine successfully operating using the crab-waist collision scheme [12] is the electron-positron collider Double Annular $\Phi$-Factory for Nice Experiments (DA $\Phi N E$ ) [13] at INFN. This collider is designed with a flexible lattice and optics, where, for example, beams are brought to collision with an optics providing a negative momentum compaction factor [14]. The flexible design of DAФNE allows also for interesting studies of various optics concepts, or studying the impact of misalignment tolerances on crab-waist collisions. In addition to testing new optics, operation of high intensity beams can be studied, as beam currents of up to $2.5 \mathrm{~A}$ and $1.4 \mathrm{~A}$ are stored, respectively, for electrons and positrons. Dynamic beam simulations for the FCC-ee and its booster reveal an extremely fast coupled bunch instability with a growth time of a few turns [15]. A powerful feedback system would hence be required, where experience from DA $\Phi$ NE will help finding the most suitable design for the FCC-ee and its booster.

FCC-ee is designed to collide polarized beams which are used for precise energy calibration, similar to VEPP-4M [16] at BINP. Although beam polarization is achieved naturally in a lepton storage ring, the polarization time at the lowest FCC-ee energy is $250 \mathrm{~h} \mathrm{[5]} \mathrm{and} \mathrm{therefore} \mathrm{elements}$ decreasing this time, such as wigglers, would need to be installed. Performing a dedicated depolarization allows for precise energy calibration, demanded for high precision physics experiments. Using this technique an energy calibration of $10^{-6}$ is achieved at VEPP-4M [17], which would correspond to $100 \mathrm{keV}$ for FCC-ee.

Performing beam studies at the Karlsruhe Research Accelerator (KARA) [18] at KIT allows studying prototypes of various components of the FCC-ee beam instrumentation. KARA is designed to allow for flexible beam parameters, such as bunch lengths of a few $\mathrm{mm}$ up to $24 \mathrm{~mm}$, a minimum bunch spacing of $2 \mathrm{~ns}$ for multi-bunch operation or beam currents of up to $200 \mathrm{~mA} \mathrm{[19].}$ Recent studies measured the longitudinal bunch profiles using an electro-optical spectral decoding combined with an ultra-fast line camera and would be also suitable for the FCC-ee, thanks to similar beam parameters [19]. 
Achieving the ambitious FCC-ee design performance demands precise optics control, emittance tuning and tight alignment tolerances. The latter are similar to the Positron-Electron TandemRing Accelerator (PETRA) III at DESY and its upgrade PETRA IV [20]. Therefore, emittance tuning procedures respecting various element and girder tolerances performed at PETRA III will help defining those tolerances for the FCC-ee [21] and the respective emittance growth from misalignments. In addition to defining tolerances, optics measurements using Turn-by-Turn (TbT) data can be performed at PETRA III, allowing also for testing dedicated mitigation strategies.

The presently highest luminosity collider is SuperKEKB [22] at KEK, where in June 2021 $3.1 \times 10^{34} \mathrm{~cm}^{-2} \mathrm{~s}^{-1}$ has been achieved [23]. For this record the crab-waist collision scheme has been applied, where the crab-waist sextupole has been powered to $80 \%$ and $40 \%$, respectively, for the $4 \mathrm{GeV}$ positron ring (LER) and the $7 \mathrm{GeV}$ electron ring (HER) [23]. Its present design goal is set to an about 20 times higher luminosity of $60 \times 10^{34} \mathrm{~cm}^{-2} \mathrm{~s}^{-1}$, aimed to be achieved around 2028 . An international task force has been established to help reaching its design, where the first optics meeting has recently taken place [24]. Similar to FCC-ee, SuperKEKB is an electron-positron collider using the crab-waist collision scheme and top-up injection. Moreover, both machines are designed with the pseudo $-I$-transformation in the arcs. One remarkable achievement of SuperKEKB is operating an optics with a $\beta_{y}^{*}$ of $0.8 \mathrm{~mm}$ in 2020 , the lowest value demanded for the FCC-ee and hence, demonstrating the feasibility of one crucial optics aspect. Thanks to numerous similarities, understanding SuperKEKB probes commissioning and operational challenges of FCCee and also helps concluding on the most suitable FCC-ee design. Although SuperKEKB design foresees a $\beta_{y}^{*}$ of $0.3 \mathrm{~mm}$, recent simulations demonstrate an optics where $\beta_{y}^{*}=0.09 \mathrm{~mm}$ [25].

Performing optics measurements at SuperKEKB allows to monitor the beam optics at various machine conditions, helps revealing possible unexpected error sources and demonstrates techniques suitable for FCC-ee. An extensive measurement campaign [26] aims at understanding the merits and limitations of TbT measurements of optics parameters such as $\beta$-functions or amplitude detuning after applying horizontal single kicks, or while driving the beam continuously during data acquisition using a Phase Lock Loop. For example, LER measurements at various bunch currents show intensity dependent effects [27], where one observation is a negative tune shift with increasing bunch intensity, as shown in Fig. 1. In this measurement the vertical intensity dependent tune shift is explained fully by known sources, whereas horizontally about $30 \%$ are not explained with the used impedance model. As the TbT data is acquired after applying a single horizontal kick, the precision in the vertical plane is poorer. A complementary optics measurement technique is $\mathrm{K}$-modulation, which is successfully used to measure $\beta_{x, y}^{*}$ for HER [28]. K-modulation also allows
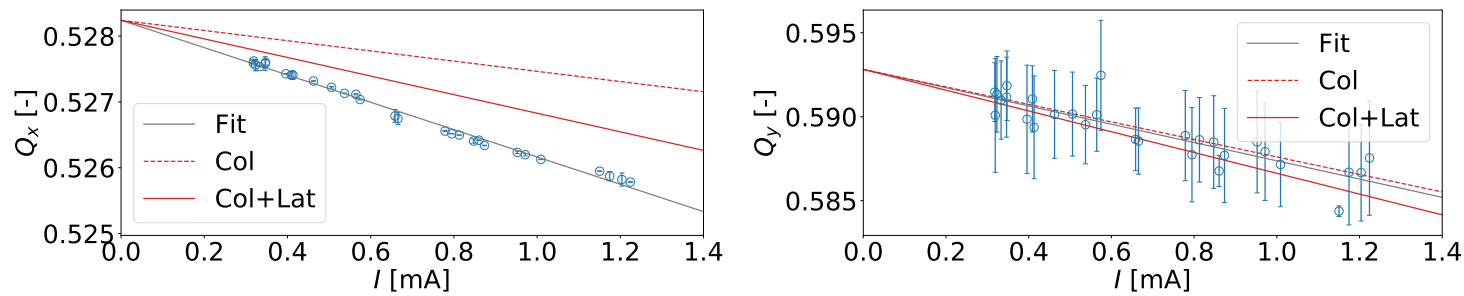

Figure 1: Measured horizontal (left) and vertical (right) tune over bunch current for SuperKEKB LER (Fit); comparison to the expected collimator contribution (Col) and additional lattice contributions (Col+Lat) [27]. 
improving the knowledge of beam dynamics close to the final focus.

\subsection{Summary and outlook}

Numerous key concepts of the FCC-ee could be demonstrated in advance at existing facilities, where various examples are given in this article. For example, the most suitable positron source could be studied at the SwissFEL, the crab-waist collision scheme at DAФNE and SuperKEKB, or spin dynamics and energy calibration at VEPP-4M. Emittance tuning techniques at PETRA III and SuperKEKB will also be applicable for the FCC-ee and beam tests at KARA will allow improving crucial beam instrumentation. As SuperKEKB shares various key concepts with FCC-ee, obtained commissioning and operational experience will influence greatly the final design choices of FCCee. Performing such dedicated beam tests will demonstrate the FCC-ee before its commissioning, foreseen around 2040 .

\section{Acknowledgments}

We would like to thank I. Agapov, I. Chaikovska, T.K. Charles, A. Drago, Y. Funakoshi, K. Furukawa, E. Gianfelice, T. Ishibashi, M. Migliorati, C. Milardi, N. Muchnoi, A.-S. Müller, G. Niehues, S. Nikitin, K. Ohmi, Y. Ohnishi, H. Sugimoto, P. Thrane, A. Wegscheider, R. Yang, and $\mathrm{M}$. Zobov for valuble commments and fruitful discussions.

\section{References}

[1] O. Brüning et al. (eds.), "LHC design report, Vol. 1 The LHC main ring", Rep. No. CERN2004-003-V-1, 2004.

[2] G. Apollinari et al. (eds.), "High-Luminosity Large Hadron Collider (HL-LHC): Technical Design Report V.0.1”, Rep. No. CERN-2017-007-M, 2017.

[3] FCC study, Accessed 26th September 2021. https://fcc . web.cern.ch/

[4] M. Benedikt, A. Blondel, P. Janot, M. Mangano, and F. Zimmermann "Future circular colliders succeeding the LHC”, in Nat. Phys. 16, pp. 402-407, 2020.

[5] M. Benedikt et al. (eds.), "Future Circular Collider study, Volume 2: The Lepton Collider (FCC-ee) Conceptual Design Report”, in Eur. Phys. J. ST. 228, pp. 261-623, 2019.

[6] M. Benedikt et al. (eds.), "Future Circular Collider study, Volume 3: The Hadron Collider (FCC-hh) Conceptual Design Report”, in Eur. Phys. J. ST. 228, pp. 755-1107, 2019.

[7] K. Oide, "Optics and layout - update and plan", FCC Week 2021, Geneva, Switzerland.

[8] B. Härer, B.J. Holzer, Y. Papaphilippou and T. Tydecks, "Status of the FCC-ee top-up booster synchrotron”, in Proc. IPAC'18, Vancouver, BC, Canada, MOPMF059, pp. 250-254, 2018.

[9] M. Mangano et al. (eds.), "Future Circular Collider Study. Volume 1: Physics Opportunities. Conceptual Design Report”, in Eur. Phys. J. ST. 79, pp. 474, 2019. 
[10] I. Chaikovska, "FCC-ee positron source ”, FCC Week 2021, Geneva, Switzerland.

[11] R. Ganter (ed.), “SwissFEL Conceptual Design Report”, PSI Bericht Nr. 10-04, 2021.

[12] M. Zobov et al., "Test of Crab-Waist collisions at the DAФNE $\Phi$ factory", in Phys. Rev. Let. 104, pp. 174801, 2010.

[13] G. Vignola, "DA $\Phi N E$, The Frascati $\Phi$-factory”, in Proc. PAC'93, Washington, DC, USA, pp. 1993-1997, 1993.

[14] M. Zobov et al., "DAФNE experience with negative momentum compaction", in Proc. EPAC'06, Edinburgh, Scotland, TUODFI02, pp. 989-921, 2006.

[15] A. Drago, "Feedback systems for FCC-ee", in Proc. eeFACT'16, Daresbury, UK, TUT3AH9, pp. 136-142, 2016.

[16] V. Anachin, et al., "VEPP-4M collider: status and planes", in Proc. EPAC'93, Stockholm, Sweden, WEP06J, pp. 400-403, 1998.

[17] A. Bogomyagkov, et al., "VEPP-4M operation at low energy”, in Proc. APAC'04, Gyeongju, Korea, THM204, pp. 536-538, 2004.

[18] E. Huttel et al., "Operation with a low emittance optics at ANKA", in Proc. PAC'05, Knoxville, USA, RPAE037, pp. 2467-2469, 2005.

[19] G. Niehues, "Longitudinal bunch-by-bunch profile measurements at KARA based on EO sampling \& developments for FCC ”, FCC Week 2021, Geneva, Switzerland.

[20] C.G. Schroer et al. (eds.), “Petra IV”, Rep. No. PUBDB-2019-03613, 2019.

[21] T.K. Charles et al., "Update on the low emittance tuning of the e+/e- future circular collider", in Proc. IPAC'21, Campinas, SP, Brazil, WEPAB011, pp. 2601-2604, 2021.

[22] K. Akai, K. Furukawa and H. Koiso, "SuperKEKB Collider”, arXiv:1809.01958v2, 2018.

[23] Y. Funakoshi, "Private communication", June 2021.

[24] 1st SKEKB Int. Task Force Optics Subgroup Meeting, 22nd September 2021. https://kds. kek.jp/event/39396

[25] P. Thrane et al., "Probing linear collider final focus systems in SuperKEKB", Rep. No. CERNACC-2017-0052, CLIC-Note-1077, 2017.

[26] J. Keintzel et al., "SuperKEKB optics measurements using turn-by-turn beam position data", in Proc. IPAC'21, Campinas, SP, Brazil, TUPAB009, pp. 1352-1355, 2021.

[27] J. Keintzel et al., "Impact of bunch current on optics measurements in SuperKEKB", in Proc. IPAC’21, Campinas, SP, Brazil, TUPAB010, pp. 1356-1359, 2021.

[28] P. Thrane et al., "Measuring $\beta^{*}$ in SuperKEKB with K-modulation", in Phys. Rev. Accel. Beams 23, pp. 012803, 2020. 